\title{
MAP2 and Tau Segregate into Dendritic and Axonal Domains After the Elaboration of Morphologically Distinct Neurites: An Immunocytochemical Study of Cultured Rat Cerebrum
}

\author{
Kenneth S. Kosik and Elizabeth A. Finch \\ Center for Neurologic Diseases, Department of Medicine, Neurology Division, Brigham and Women's Hospital, Boston, \\ Massachusetts 02115
}

\begin{abstract}
We sought to determine whether the strict segregation of MAP2 and tau into somatodendritic and axonal compartments in situ was maintained in dissociated neuronal cultures of the rat cerebrum. Cultures grown under serum-free conditions were immunolabeled with monoclonal antibodies specific for MAP2 and tau. At $14 \mathrm{~d}$ after plating, a clear distinction between MAP2- and tau-immunoreactive neurites was apparent. MAP2-immunoreactive neurites were relatively short, thick, tapering, and branched. Tau-immunoreactive neurites formed a crisscrossing meshwork of long, fine-caliber neurites, which, in more densely plated cultures, had a tendency to form thick, ropelike fascicles. Unlike the MAP2 pattern, tau antibodies labeled somata only lightly. Since distinct populations of neurites were labeled with the 2 antibodies, we sought to observe the development of the topographically distinct compartments by double-labeled immunocytochemistry with both polyclonal and monoclonal antibodies to MAP2 and tau. Cells observed within the first $8 \mathrm{hr}$ after plating demonstrated equally intense MAP2 and tau immunoreactivity in a coextensive distribution throughout the cell body and initial neurites. By $16 \mathrm{hr}$, some neurites began to assume dendritic and axonal features; however, many such processes contained reaction product for both MAP2 and tau. Beginning at this time, neurites that appeared axonal showed a progressively weaker reaction with MAP2 antibodies, and neurites that appeared dendritic showed a progressively weaker reaction with tau antibodies. In most neurites the diminution appeared to occur uniformly over the entire extent of the neurite. During this transformation period there were occasional axon-like neurites that contained MAP2 immunoreactivity proximally, while tau immunoreactivity extended over the entire length of the neurite. We conclude that neurons in culture are able to compartmentalize MAP2 and tau into their appropriate processes and only attain an apparently homogeneous population of one of these MAPs after the neuron has assumed dendritic and axonal features. The analysis also lends indirect support to the hypothesis that microtubule-associated proteins (MAPs) form this association at the distal extent of the growing neurite.
\end{abstract}

\footnotetext{
Received Dec. 8, 1986; revised Apr. 22, 1987; accepted Apr. 23, 1987.

This work was supported by NIH Grants 5 R01 AG06172 and 5 K07 NS00835 (K.S.K.).

Correspondence should be addressed to Kenneth S. Kosik, M.D., Center for Neurologic Diseases, Department of Medicine, Neurology Division, Brigham and Women's Hospital, 75 Francis Street, Thorn 1226, Boston, MA 02115.

Copyright @ 1987 Society for Neuroscience $0270-6474 / 87 / 103142-12 \$ 02.00 / 0$
}

The neuron is a highly polarized cell that develops, early in the course of its differentiation, a fundamental asymmetry, recognized as axonal and dendritic processes. These processes show numerous morphological and electrophysiological distinctions.

A strict division between the proximal portion of the dendrite and the perikaryon is not possible either biochemically or morphologically. Macrostructural features of the dendrite are as follows: Dendrites taper gently along the course of their projection from the cell body, with the length of the dendritic process proportional to the diameter of the stcm. Dendritic processes contain structures such as spines along their course, in contrast to axons, which show distensions only at their termini. Dendrites tend to branch at acute angles, whereas axonal branches are usually orthogonal or obtuse. Dendritic branches, unlike axons, are thinner than their parent stem and distribute their mass unequally between their branches. The minimal diameter of the most distal portion of a dendrite ranges from 0.5 to 1.0 $\mu \mathrm{m}$, whereas unmyelinated axons can be as thin as $0.1-0.15 \mu \mathrm{m}$ (Bartlett and Banker, 1984b). The cytoskeletal ultrastructure also suggests differences, in that microtubules are generally distributed uniformly throughout dendrites, and there is a relative paucity of neurofilaments. Axons, on the other hand, often have a dense array of neurofilaments, with their microtubules arranged in small clusters among the neurofilaments. The ratios among the component polypeptides of the neurofilament triplet also differ in axons and dendrites (Shaw et al., 1981; Hirokawa et al., 1984). Many of the fundamental features of axons and dendrites have been reproduced in neuronal cell culture systems (Landis, 1977; Neale et al., 1978; Wakshull et al., 1979; Kriegstein and Dichter, 1983). The ability to express well-formed axons and dendrites is thought to arise intrinsically, because cultured neurons can develop the features of these processes when devoid of contact with other neurons (Bartlett and Banker, 1984a, b). Studies by Bartlett and Banker (1984a, b) have convincingly demonstrated that neurites with the light-microscopic appearance of dendrites contain ribosomes, particularly at branch points, and maintain their functional polarity with regard to the correct deployment of pre- and postsynaptic elements. These studies permit the conclusion that neurites with the light-microscopic morphological features of an axon or dendrite can, in general, be assumed to show the ultrastructural features of an axon or a dendrite.

Recently, cytoskeletal proteins that also show strict somatodendritic and axonal compartmentalization within the neuron have been partially characterized. The microtubule-associated proteins (MAPs), tau and MAP2, may, in part, define axonal 
and somatodendritic microtubule domains, respectively (Matus et al., 1981; Miller et al., 1982; Tytell et al., 1984; Binder et al., 1985). The topographical segregation of these molecules is also maintained in culture systems. In explants of superior cervical ganglia, MAP2 immunoreactivity was confined to the dendriterich region of the cell body mass, and upwards of $85 \%$ of the tau immunoreactivity was found in the axon-rich region (Peng et al., 1986). In cultured hippocampal neurons MAP2 immunoreactivity was confined to the somatodendritic compartment and excluded from the dense plexus of fine-caliber axonal processes (Caceres et al., 1984). When neurons from this same culture system were observed developing processes in vitro, MAP2 immunoreactivity was present in axon-like processes during the first week of neurite formation, after which time it disappeared (Caceres et al., 1986).

We have studied neurite formation in dissociated rat cultures from the cerebral cortex with regard to the development of putative axons and dendrites and their progressive segregation of tau and MAP 2 immunoreactivity into distinct compartments.

\section{Materials and Methods}

Production of cell cultures. The cerebral cortex was dissected from embryonic day 18 rats. The tissue was prepared for culture using defined media according to the methods of Bankcr and Cowan (1977) and Bartlett and Banker (1984a), modified only by the addition of $0.3 \mu \mathrm{g} /$ $\mathrm{cm}^{2}$ laminin (Collaborative Research, Lexington, MA) to $35 \mathrm{~mm}$ polylysine-treated culture dishes. Plating densities ranged from $0.5 \times 10^{5}$ to $3 \times 10^{\mathrm{s}}$ cells $/ \mathrm{cm}^{2}$. Some cells were plated on coverslips and also treated with polylysine and laminin. Regions immediately adjacent to the coverslip grew as a low-density culture, often permitting the visualization of neuronal processes in isolation. Cultures were fixed for immunocytochemical analysis at the following time points: $3,6,16,24$, 48 , and $72 \mathrm{hr}$, and 7 and $14 \mathrm{~d}$. Astroglial cultures were prepared from postnatal day 1 rat cortex by the method of McCarthy and de Vellis (1980) and fixed after $14 \mathrm{~d}$.

Monoclonal and polyclonal antibodies. The preparation of the monoclonal and polyclonal antibodies to tau and MAP2 has been described previously (Kosik ct al., 1984, 1986; Escobar et al., 1986; Galloway et al., 1987). These antibodies were tested for specificity on immunoblots of microtubule preparations and total cortical homogenates, according to the method of Towbin et al. (1979). They consisted of the following: (1) monoclonals $5 \mathrm{F9}$ and 4F7, raised against twice-cycled rat brain microtubules, specific for 2 distinct epitopes within MAP2; (2) monoclonal $5 \mathrm{E} 2$ raised against human fetal tau protein, specific for both fetal and adult tau proteins; (3) polyclonal R4 raised against rat brain MAP2, specific for MAP2 (the generous gift of Dr. Enrique Silva); (4) polyclonal anti-tau raised against SDS-extracted Alzheimer paired helical filaments, specific for both fetal and adult tau proteins (Kosik et al., 1986). Anti-galactocerebroside was kindly provided by Dr. Ranscht (Ranscht et al., 1982).

Immunocytochemical analyses. Cultures were prepared for immunocytochemistry by fixing the cclls for $30 \mathrm{~min}$ with periodate, lysine, and paraformaldehyde, as described by McLean and Nakane (1974). Blocking with $10 \%$ goat serum and $0.2 \%$ Triton X-100 in Tris-saline was done at room temperature for $1 \mathrm{hr}$. Cultures were incubated with the primary antibodies listed above in $1 \%$ goat serum overnight at $4^{\circ} \mathrm{C}$ and for $2 \mathrm{hr}$ at room temperature the next morning. Monoclonal antibodies were ammonium sulfate-precipitated supernatants rehydrated at a 4-fold concentration and used at a dilution of 1:20. Polyclonal antibodies were used at 1:500. Following three $5 \mathrm{~min}$ washes with Trissaline the cultures were immunolabeled by the ABC biotin-avidin method (Hsu et al., 1981) according to the instructions in the Vectastain (Vector Laboratories, Burlingame, CA) package insert. Biotinylated affinity-purified anti-mouse $\operatorname{IgG}$ to both heavy and light chains was used as the secondary antibody. The reaction product was visualized with freshly prepared $0.1 \%$ diaminobcnzidinc tetrahydrochloride and $0.02 \%$ hydrogen peroxide in 0.1 Tris buffer, $\mathrm{pH}$ 7.2. For double-labeling, a polyclonal anti-tau was incubated together with a monoclonal antiMAP2, and the results were confirmed by replicating the distribution of reaction product with a monoclonal anti-tau used in combination with a polyclonal anti-MAP2. The double-labeling experiments were visualized with fluorescein- or rhodamine-conjugated antibody $(1: 25)$ to either mouse or rabbit $\operatorname{IgG}$, as appropriate. These secondary antibodies were added simultaneously to the cultures and incubated in the dark, as described above. Control experiments included reversing the fluorescein- and rhodamine-conjugated antibodies, as well as treatment with either pre-immune antisera or NS-1 supernatants for all antibodies.

\section{Results}

\section{Distribution of tau and MAP2 in $14 d$ cultures}

Examination of the $14 \mathrm{~d}$ rat cortical cultures by phase-contrast microscopy suggested the presence of a multiplicity of neuronal morphologies that were reminiscent of pyramidal, stellate, and polymorphic cclls, as well as glial and fibroblastic elements. Occasional cell aggregates were present, depending on the plating density, and by $14 \mathrm{~d}$ these aggregates were connected by thick fascicles. Neurites with axonal and dendritic morphologies, as described by Bartlett and Banker (1984a), were apparent. Both the monoclonal antibodies to MAP2 and the MAP2 polyclonal antibody stained dendrite-like processes that were thick, lapering, and relatively short, with acute-angle branches (Fig. 1C). Some dendritic arbors consisted of multiple higher-order branches extending more than $200 \mu \mathrm{m}$ from the cell body. Terminal dendritic fields consisted of highly ramified thick processes, approximately $0.6 \mu \mathrm{m}$, that were stained even at their most distal extensions. Multiple small lateral protrusions were detected along some MAP2-immunoreactive processes, and appeared more abundant during the first week than in more mature cultures (Fig. 2A).

Cultures stained with either polyclonal or monoclonal antibodies to tau revealed a markedly different image of immunoreactive processes. The distinct populations of neurites stained with the tau and MAP2 antibodies clearly demonstrated the separate compartmentation of these molecules in the culture system. Most prominently stained was a fine-caliber background meshwork of processes that looped and crisscrossed over the dish (Fig. 1, $A, B$ ). These processes had a tendency to form bundles that usually connected cellular aggregates in a ropelike fashion (Fig. 1 $A$ ). In contrast to brain tissue sections from either rat (Binder et al., 1985) or human (Kowall and Kosik, 1986), some perikarya contained tau immunoreactivity. Not every neuronal cell body stained, and those cell bodies that did stain were considerably less dense than those in the more immature cultures (see below). To be sure that our tau antibodies did not recognize a tau epitope that was present in cell bodies of rat brain tissue sections, we perfused an adult rat with paraformaldehyde-lysine-periodate (McLean and Nakane, 1974) and demonstrated an axonal pattern with $5 \mathrm{E} 2$ that replicated the findings of Binder et al. (1985). Such sections demonstrated an axonal pattern without evidence of non-neuronal cell bodies staining in the white matter or of neuronal cell bodies staining in the gray matter (J. E. Crandall and K. S. Kosik, unpublished observations). The presence of distinct MAP2 and tau domains was not, however, absolute. Although occasional neurites that appeared dendritic stained with tau antibodies (see Fig. $5 B$ ), the vast majority of such neurites were not tau-immunoreactive. Features that might complicate the interpretation of a neurite as being either a dendrite or an axon are (1) small-caliber axonal fascicles that are thickened proximally and narrow as individual fibers peel off; (2) axons that arise from dendrites; and (3) axons that course along with a dendrite. Thus, an unequivocal discrimination between axons and dendrites is probably not possible in every instance. Some neurons had more than one tau-positive 

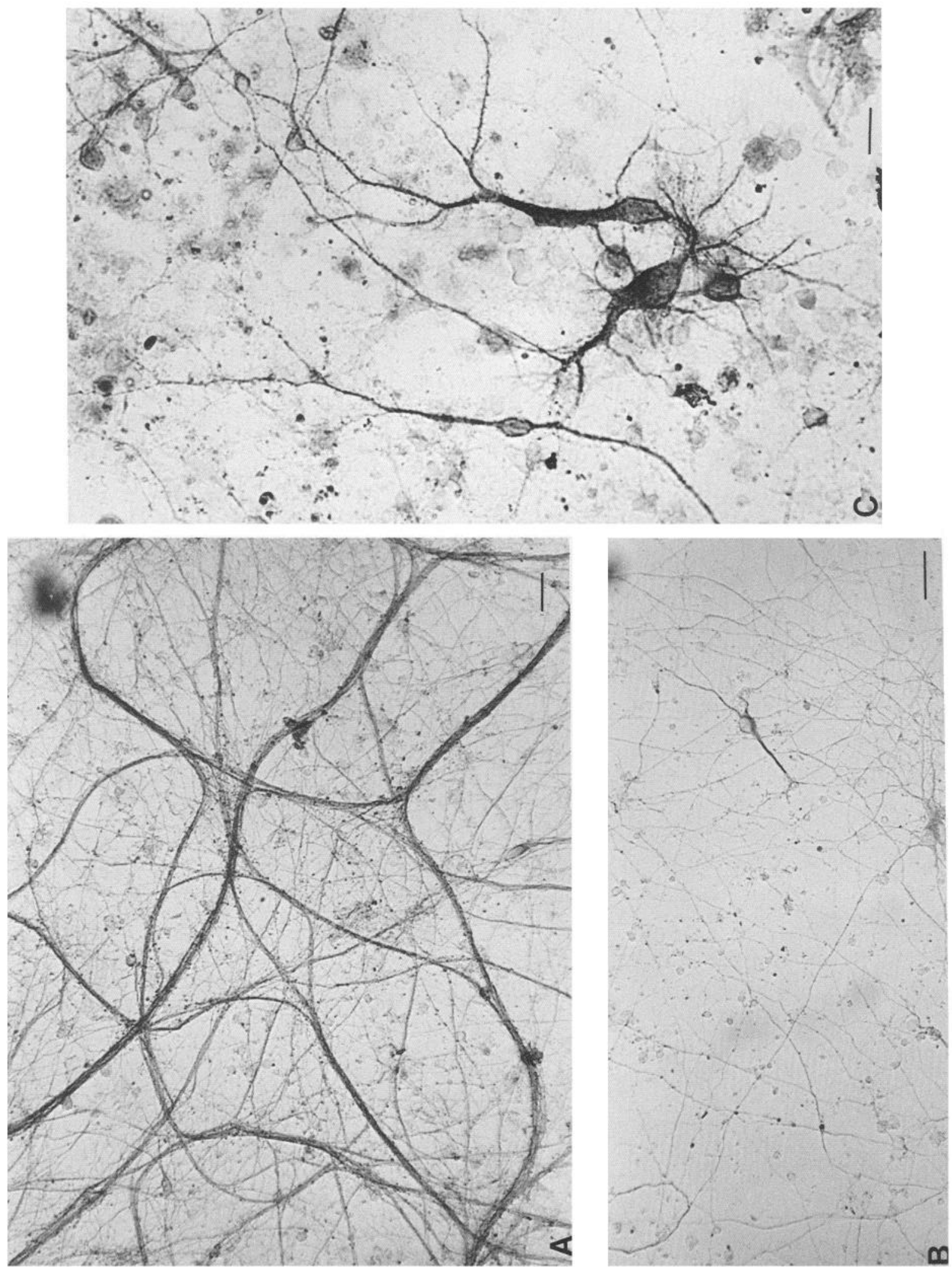

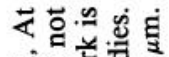

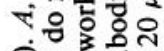

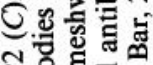

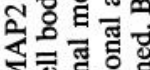

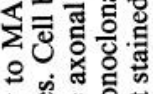
공.

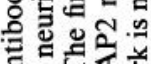
ส बु.

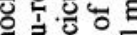

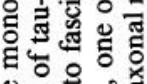
झ눙형

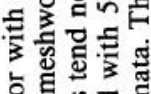
Qิ उत 크의 염 흘 त这它

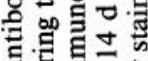
웡

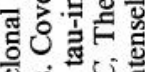
过记 융 छํํㄹ 通 $\exists 0$.

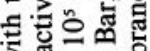
$3 \times$

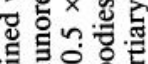

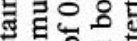
क. 궁 का 可 동월 包 可高尔 才必公圆 उ

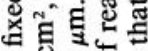
충ㅇㅇ 롱 능 赵记 × 的.

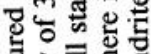
政 过券 $-200$

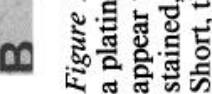



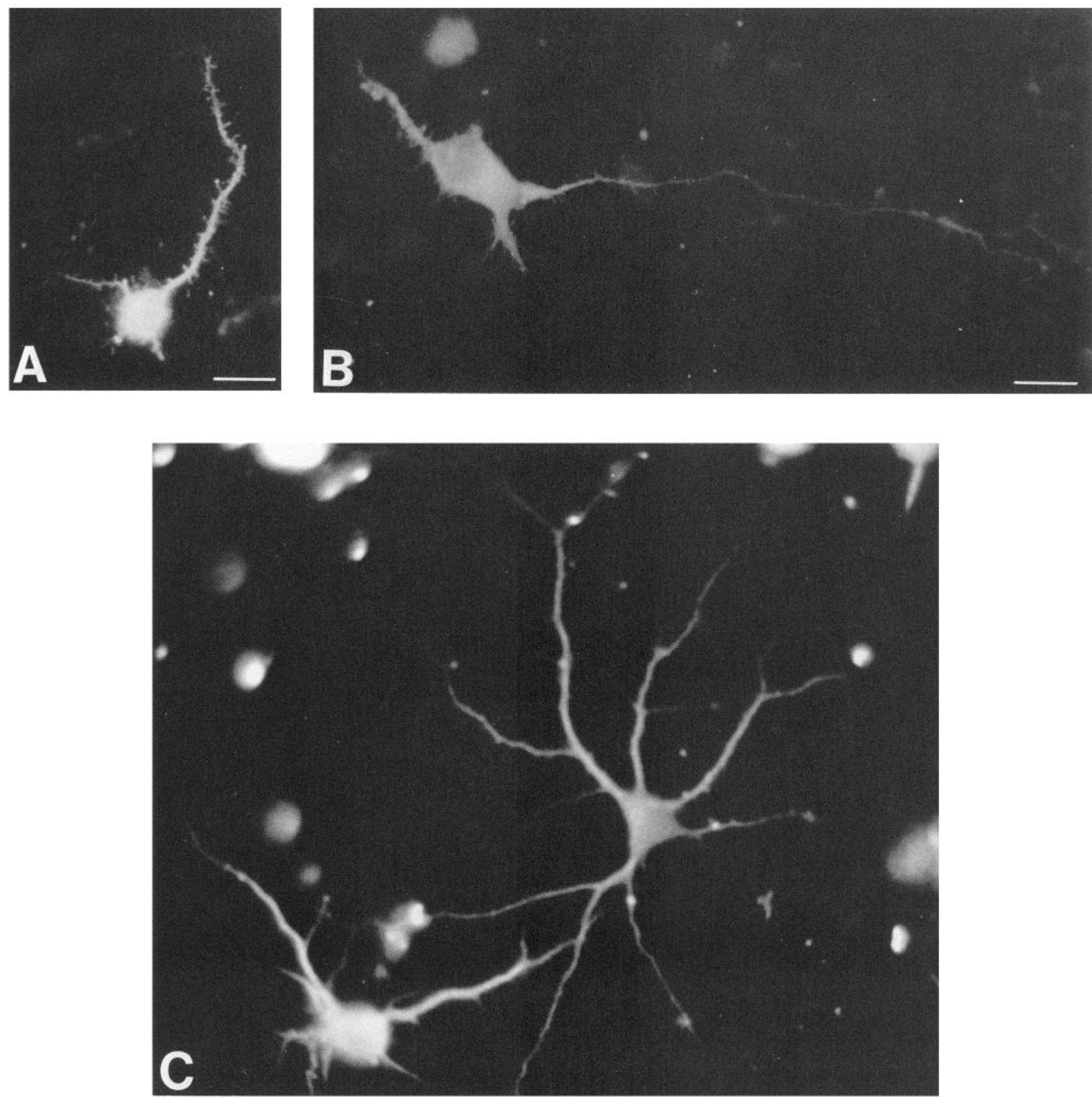

Figure 2. Cultured rat cerebrum fixed $72 \mathrm{hr}$ after plating, stained with the monoclonal antibody to MAP2, 5F9, and visualized with fluoresceinconjugated secondary antibody. $A$, The longest neurite on this neuron has begun to assume some dendritic features. Bar, $20 \mu \mathrm{m}$. $B, \mathrm{~A}$ neuron that has elaborated 2 short, thick neurites and a long, thin neurite that does not branch until its terminal extension. Despite the axonal features of the long neurite, it is MAP2-immunoreactive at this early time point. Bar, $20 \mu \mathrm{m}$. $C$, A neuron that has attained a more complex appearance, including tertiary branches that exhibit a dendritic morphology. Bar, $20 \mu \mathrm{m}$.

process, although a single neurite, sometimes emanating from the proximal stem of the putative dendrite, was often more intensely stained than any of the other tau-positive processes.

Unlike the MAP2 stained cultures, some tau-immunoreactive cells had a non-neuronal appearance. To determine whether non-neuronal cells in culture expressed tau, glial cultures were prepared from postnatal day 1 rats. The bed of astrocytes was clearly negative with the tau antibodies. Phase-dark cells with the appearance of oligodendrocytes (Fig. 3) were lightly stained by anti-tau, but were negative with MAP2. To demonstrate that these phase-dark cells were oligodendrocytes, the cultures were double-stained with the specific marker, anti-galactocerebroside and anti-tau (Fig. 3). Light staining with tau was evident in the oligodendrocytes. MAP2 antibodies did not stain any cell types in the glial culture.

\section{Double-staining with tau and MAP2 antibodies}

To determine the extent to which tau and MAP2 colocalize, double-staining with the 2 antibodies was performed. In $14 \mathrm{~d}$ cultures, neurites generally reacted with either the MAP2 or the 


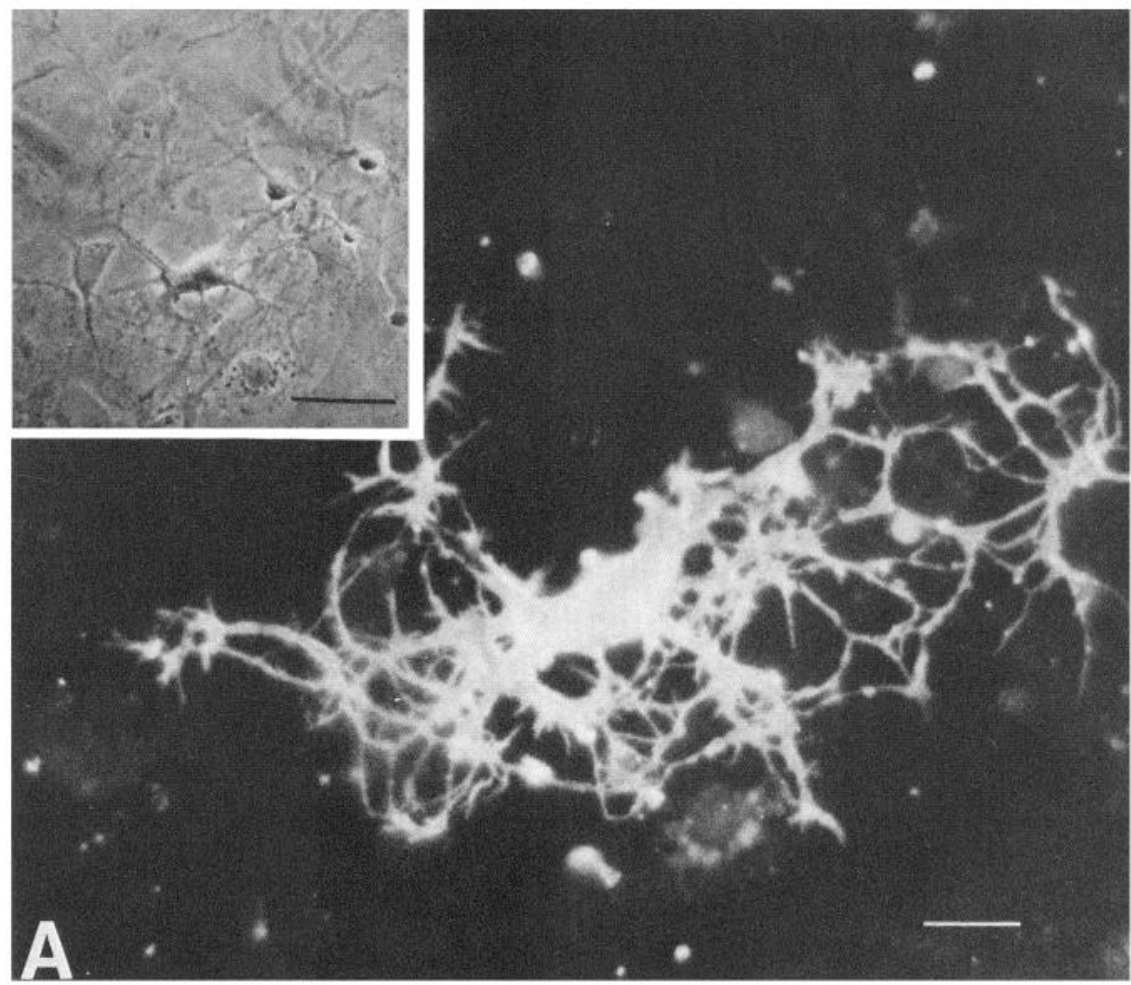

Figure 3. Astrocyte culture from postnatal day 1 rat cerebrum double-labeled with anti-galactocerebroside $(A)$ and anti-tau $(B)$. Inset shows the same field by phase-contrast microscopy, in which the phase-dark cell is identified as an oligodendrocyte by the presence of galactocerebroside. The same cell is also labeled by the tau antibody $(B)$. Tau immunoreactivity in oligodendrocytes was less intense than in neurons, but above the background absence of reaction product in astrocytes. The bright spots in $B$ are artifact. $B a r(A, B), 20 \mu \mathrm{m}$. Bar (inset), $50 \mu \mathrm{m}$.

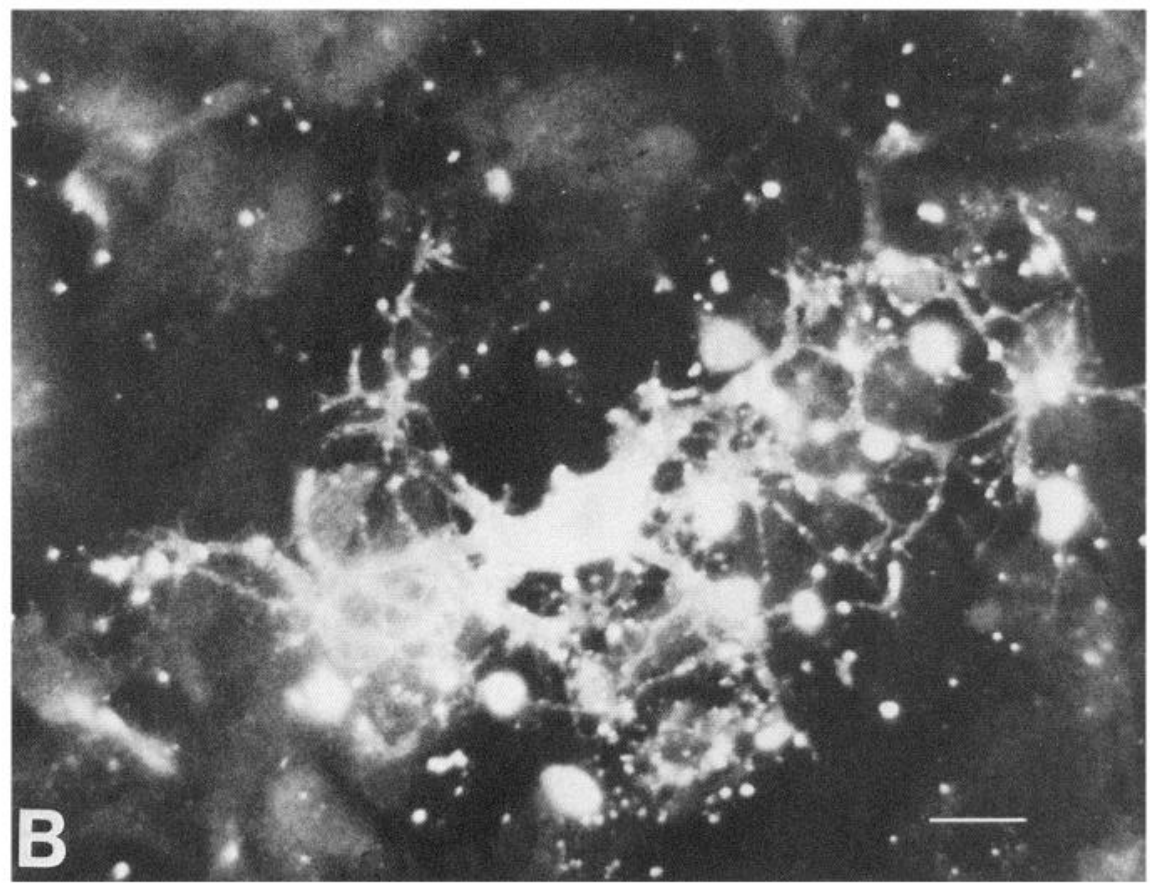

tau antibody in a dendritic or axonal pattern, respectively. An individual MAP2-positive fiber could sometimes be detected coursing within the thick tau-positive fascicles. Occasional neurites stained over their entire course with both antibodies. Thus, tau and MAP2 coexisted in the same neuronal process, although tau immunoreactivity was more intense in those neurites with an axonal appearance and MAP2 immunoreactivity was more intense in those with a dendritic appearance. Neurites with axonal features were observed in which the MAP2 immunoreac- tivity was limited to the proximal portion of the neurite, while the tau immunoreactivity extended over the entire neurite. Some of these neurites may have been axons that arose from dendrites. Other such processes may have represented an intermediate stage during which either MAP2 or tau was eliminated from the developing neurite (see below).

As proof that the MAP2 monoclonal, 5F9, gave the same signal as the MAP2 polyclonal antibody, double-staining with these antibodies showed identical patterns. Similarly the tau 

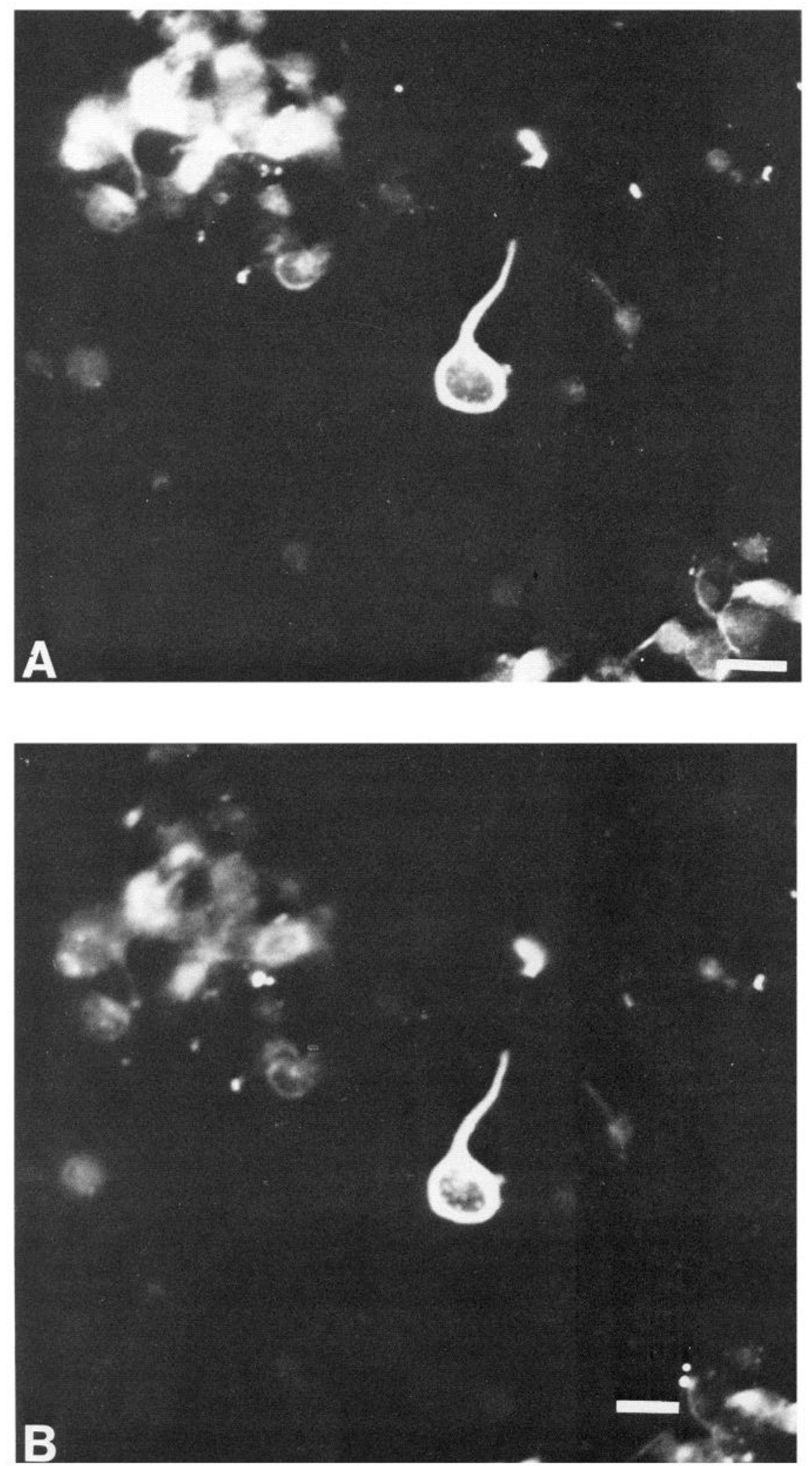

Figure 4. Cultured rat cerebrum fixed $8 \mathrm{hr}$ after plating and double-stained with the MAP2 polyclonal antibody $(A)$ and with the tau monoclonal antibody $(B)$. The cell body and the initial extension of the neurite are intensely stained with both antibodies. Bars, $25 \mu \mathrm{m}$.

monoclonal, 5E2, was coextensive with the tau polyclonal. The polyclonal antibodies consistently gave a stronger signal; therefore, the segregation of MAP2 and tau was confirmed with both polyclonal and monoclonal antibodies to each of these proteins (see Materials and Methods). Any distinctions between trace amounts of a particular antigen and its complete absence were not possible with these nonquantitative immunocytochemical techniques. 

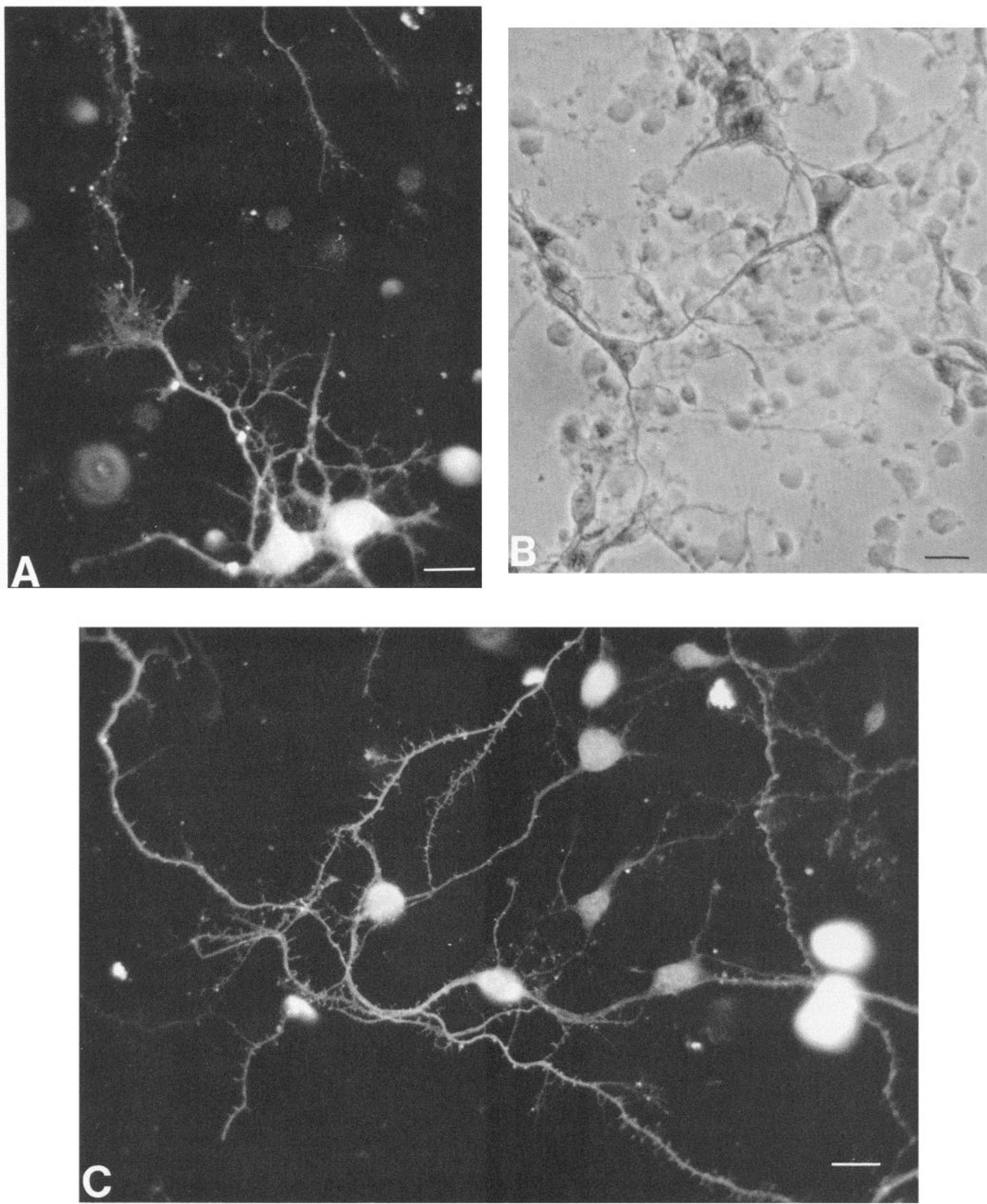

Figure 5. Cultured rat cerebrum fixed $72 \mathrm{hr}$ after plating and stained with the monoclonal antibody to tau. $A$, Two adjacent neurons elaborating a complex array of growth cone morphologies, as has been observed in neurite migration over non-neural substrates. Fluorescein-labeled tau immunoreactivity extends into the filopodia and lamellipodia. Bar, $20 \mu \mathrm{m}$. B. During early neurite elaboration, tau immunoreactivity can be detected in 2 triangular neurons in the cell body and extending into several thickened neurites, some of which have a dendrite-like appearance. Some of the nonreactive cells in the background are probably astrocytes. The chromagen is diaminobenzidine. Bar, $20 \mu \mathrm{m}$. $C$, Fluorescein-labeled tau immunoreactivity in a more axon-like pattern, taken from another field within the same culture as $A$. Cell bodies are densely stained and some neurites contain immunoreactive filopodia that extend nearly $7 \mu \mathrm{m}$. Bar, $20 \mu \mathrm{m}$. 
The establishment of tau and MAP2 domains in neuronal culture

Both MAP2 and tau immunoreactivity were readily detectable at the earliest time point of $3 \mathrm{hr}$ after plating. This finding is consistent with the early expression of MAP2 in developing rodent cortex (Crandall et al., 1986) and the detection of tau bands on immunoblots from fetal rat brain (Kosik et al., 1986). Immunoreactivity for both antigens was coextensive in these rounded cells, most of which were without neurites. Occasional asymmetric cells could be detected, some of which had begun to elaborate a neurite. These initial neurites were consistently positive for both tau and MAP2 (Fig. 4). By $16 \mathrm{hr}$, many cells had extended short, thick processes that tapered. These dendrite-like processes were intensely MAP2-immunoreactive and, with varying degrees of intensity, were tau-immunoreactive. Some cells at $16 \mathrm{hr}$ extended long, nontapering, unbranched processes, which stained intensely with tau antibodies but only lightly with MAP2 antibodies. Cells that displayed axon-like processes extending over as much as $200 \mu \mathrm{m}$ at these early time points often had a considerably less elaborate dendritic tree. As a whole, neither axon- nor dendrite-like processes were more prominent as the cultures became established.

Many of the neurites, during the early stages of in vitro development, had prominent filopodia along their course. These protrusions were well stained with the antibodies in a fashion consistent with the staining pattern of the neurite from which they protruded. The use of Triton raises the possibility that reaction product diffused into the protrusions. Reaction product appropriate to the axonal or dendritic character of the neurite was detected within growth cones and their protrusions. Growth cones consisting of digitate filopodia and sheetlike lamellipodia varied greatly in size and complexity (Fig. $5 A$ ).

From as early as 8-16 hr in culture, neurites could be crudely classed into axon- or dendrite-like neurites, depending on their tendency to taper and their degree of branching. Throughout the 2 week observation period, neurites that appeared axonal were progressively less well stained with MAP2 antibodies, and neurites that appeared dendritic were progressively less well stained with tau antibodies. While $14 \mathrm{~d}$ cultures showed nearly complete segregation of tau and MAP2 (Figs. 1, $A-C$; 7), less mature cultures had both antigens present within a single process. At the earliest time points, when the first neurites became apparent, staining for both MAP2 and tau was intense and coextensive (Fig. 4). As cultures developed features of axons and dendrites, a dissociation emerged, with tau more intense in axon-like processes and MAP2 more intense in dendrite-like processes; but both antigens were present. Axonal and dendritic features were thus able to develop in the presence of both antigens within a single neurite. The observation of large numbers of cells at discrete time points suggested a gradual diminution of one antigen in favor of the other over the entire length of the neurite, without any apparent proximal-distal gradient in the segregation of tau and MAP2 immunoreactivity. A minority of the neurites, usually with axonal features, did show a gradient separation between MAP2 and tau. These axon-like neurites were MAP2-immunoreactive proximally and tau-immunoreactive along their entire course (Figs. 6, 7). Dendrite-like neurites with a reverse appearance were not sufficiently frequent to be the basis of a confident observation. Neurons with the gradient effect could be observed in a subset of neurons from as early as $16 \mathrm{hr}$ to $14 \mathrm{~d}$ (Figs. 6, 7).
The segregation of MAP2 and tau does not occur at the initiation of process formation, nor is their complete segregation contingent on the expression of a dendritic or axonal morphology. It therefore seems likely that both dendrites and axons can achieve many of their shape constraints and branching patterns without a complete separation of these molecules.

\section{Discussion}

Because MAP2 and tau are so clearly compartmentalized in the mature animal into a somatodendritic and an axonal domain, respectively, the developmental process by which these molecules sort may reflect upon the ontogeny of neuronal polarization. We have previously shown that MAP2 is first expressed in mouse cerebral cortex at embryonic day 14 (Crandall et al., 1986). Migrating cortical neurons did not contain MAP2 in their leading or trailing processes; the onset of MAP2 expression was apparently synchronous with the achievement of architectonic destiny and thus first appears in a laminar pattern within the subplate (Crandall et al., 1986). Although the precise onset of tau expression in rodent cortex has not been determined, it is also present before birth (Drubin et al., 1984; Kosik et al., 1986). Both tau (Mareck et al., 1980; Francon et al., 1982; Drubin et al., 1984) and MAP2 (Binder et al., 1984; Burgoyne and Cumming, 1984) undergo additional biochemical modifications during postnatal development that result in the more complex electrophoretic patterns of these proteins.

These studies demonstrate that tau and MAP2 segregate into axonal and dendritic domains in culture. However, the strict compartmentalization of these molecules seen in tissue is not observed during the elaboration of morphologically distinct neurites in culture. During maturation, tau immunoreactivity can be detected in perikarya and in some processes with a dendritic morphology, just as MAP2 immunoreactivity can be detected in axon-like processes. Thus, a pure population of the heat-stable MAPs, tau and MAP2, is not essential for the achievement in culture of at least some of the morphological features of a dendrite or axon.

The segregation of MAP2 and tau is not coincident with the elaboration of processes, but occurs after neurites have acquired some resemblance to their ultimate morphology. However, when the cultures are plated at embryonic day 18, MAP2 and tau are already expressed in situ, particularly in postmigratory neurons. Therefore, early "development" in culture represents, in part, regeneration of tau- and MAP2-immunoreactive neurites that had been sheared during dissociation. Only later, within an established, complex, neural milieu, might the in vitro paradigm recapitulate, in some measure, in vivo development. Neuronal cell bodies contain tau even at the earliest time point studied, $3 \mathrm{hr}$.

Whether tau is present at embryonic day 18 in neuronal perikarya in tissue sections is currently under investigation. A distinction from the in situ distribution of MAP2 is its presence in axon-like neurites; this was not observed during the in situ development of rodent cortex (Crandall et al., 1986). Tau remained present in neuronal cell bodies even at the longest time point studied ( 2 weeks), although the intensity of cell body staining was reduced in the more mature cultures (Fig. 7). Cultures harvested after 2 weeks continued to show the immature configuration of tau on immunoblots and did not differ from those at earlier time points (K. S. Kosik and E. A. Finch, unpublished observations). Thus, in agreement with Couchie et al. (1986), the expression of mature forms of tau does not correlate, in 

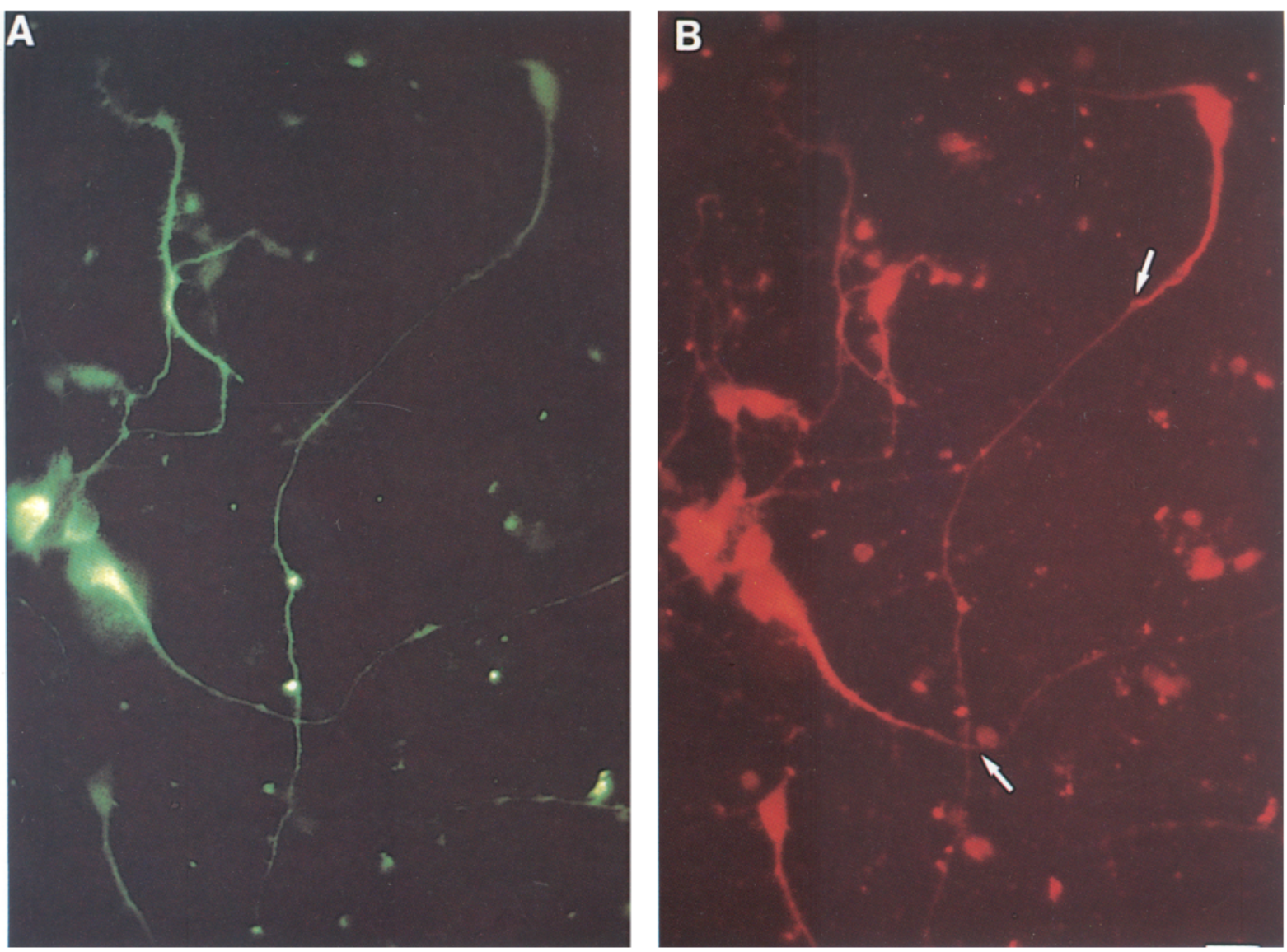

Figure 6. Cultured rat cerebrum fixed $16 \mathrm{hr}$ after plating and double-labeled with polyclonal anti-tau in $A$ and monoclonal anti-MAP2 (5F9) in $B$. Tau antibody was detected with fluorescein-conjugated anti-rabbit IgG, and MAP2 antibody was detected with rhodamine-conjugated antimouse IgG. Arrows in $B$ indicate the points on 2 different neurites where MAP2 immunoreactivity becomes less intense. At points distal to the arrows, the corresponding neurites in $A$ continue to show nearly uniform tau immunoreactivity over their entire lengths. This axon-like neurite may be eliminating MAP2 as it matures. Bar, $25 \mu \mathrm{m}$.

culture, with the appropriate segregation of tau into axon-like neurites.

The molecular level at which tau and MAP2 segregate into their appropriate processes is a matter of interest. Some data suggest a nonrandom distribution of the ribosomes that synthesize the cytoskeletal proteins tubulin, actin, and vimentin (Lawrence and Singer, 1986). The extent to which ribosomal elements, in combination with the synthesized protein, participate in achieving the correct MAP2 and tau topography is unknown. Perikaryal synthesis of tau requires that the protein be transported through the cell body and, in some cases, through the proximal portion of the dendrite (in those cells in which the axon is deployed from the proximal portion of the dendrite) until it enters the axon. If perikaryal levels are kept low, tau must move through the cell more quickly than it moves along the axon. In so doing, tau may bind microtubules when it is about to enter the axon and move through the cell body unassociated with microtubules. Alternatively, tau may be antigenically modified once it reaches the axon and only at that point become detectable.

Owing to the cohesive nature of the cytoskeleton, the maintenance of established neuronal processes requires that the choice of transporting tau to an axon or MAP2 to a dendrite be made within the perikaryon. The sorting of tau and MAP2 could be effected by the attachment of these molecules to selective subsets of microtubules within microtubule-organizing centers distinct for axonal and dendritic microtubule populations. During the establishment of dendrites and axons, sorting and elimination of microtubule subpopulations, or pruning of individual microtubules until a homogeneous population of MAPs is achieved, many occur within the process itself. This is because tau and MAP2 can colocalize in dendrite- and axon-like processes during in vitro development. One of the questions in developmental neurobiology is whether individual microtubules in the growing axon elongate at their proximal or distal end. The case for proximal assembly is supported by slow axonal transport studies (Heriot et al., 1985), whereas distal assembly is suggested by the presence of the fast-growing end of the tubule at the distal end (Heidemann et al., 1981) and the sensitivity of growth cone microtubules to Colcemid (Bamburg et al., 1986).

The latter hypothesis suggests that unassembled tubulin is transported to the growth cone for assembly, and it is likely that this pool of tubulin molecules is transported at a different rate from assembled tubulin. We have attempted to determine whether the development of distinct MAP populations within neurites occurs along a gradient, or whether the entire process gradually excludes MAP2 or tau. The work of Caceres et al. (1986) with rat hippocampal cultures describes the progressive loss of MAP2 immunoreactivity in a proximodistal fashion during in vitro development of an axon. One implication of this 

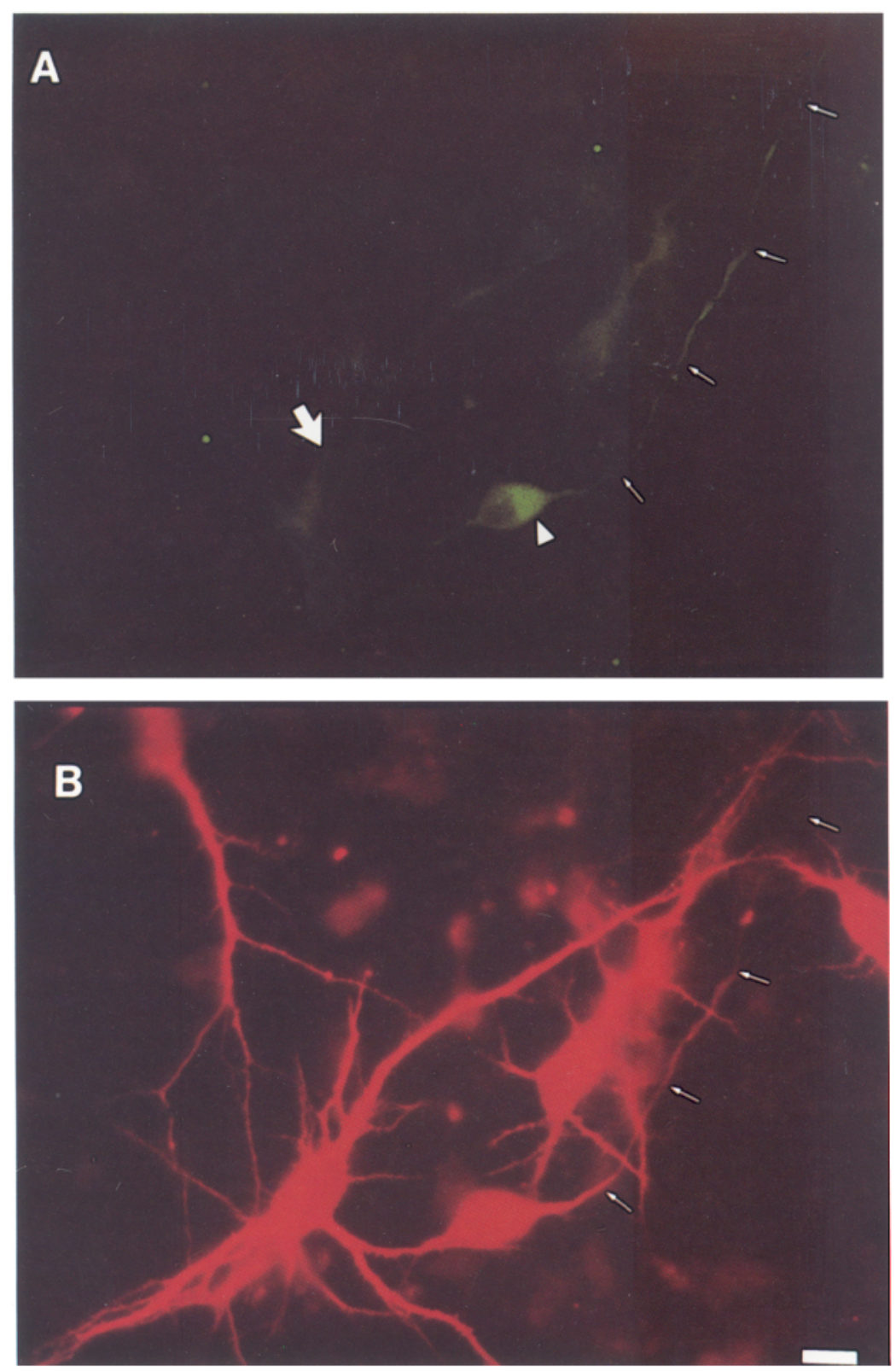

Figure 7. Cultured rat cerebrum fixed $14 \mathrm{~d}$ after plating and double-labeled with monoclonal anti-tau in $A$ and polyclonal anti-MAP2 in $B$. Tau antibody was detected with fluoresceinconjugated anti-mouse IgG, and MAP2 antibody was detected with rhodamineconjugated anti-rabbit IgG. The large arrow in $A$ demonstrates the nearly complete absence of tau reaction product within a cell body that is intensely labeled by the MAP2 antibody in $B$. The loss of somatic staining with tau antibodies is typical of the more mature cultures, as is the absence of the dendrite-like neurites seen only in $B$. The arrowhead in $A$ demonstrates the displacement of tau immunoreactivity toward a neurite with an axonal morphology. This neurite in $A$ is tauimmunoreactive nearly to the edge of the microscopic field, as indicated by the small arrows. The same neurite is indicated in $B$, where MAP2 immunoreactivity is markedly reduced or absent beyond the third small arrow, even though the polyclonal antibody used to detect MAP2 overestimates the amount of MAP2 antigen relative to the monoclonal anti-tau in $A$. Bar, $25 \mu \mathrm{m}$. observation is that microtubules elongate at their proximal end in the growing axon since newly assembled microtubules that contain tau will continue to move into the axon at a point in development when MAP2 will be prevented from entering the axon, thus giving rise to distal MAP2 immunoreactivity (Fig. 8). We have observed the elimination of MAP2 from axon-like neurites and the elimination of tau from dendrite-like neurites in a diffuse fashion. This finding is more consistent with the transport of tau, MAP2, and perhaps tubulin to the growth cone in an unassembled form, and the addition of formed microtubules at the growth cone. Some cells did display a gradient pattern in the distribution of the MAP2 and tau antigens, particularly in axon-like neurites. These neurites showed MAP2 immunoreactivity proximally and tau immunoreactivity distally (Figs. 6, 7). The gradient pattern was present in a minority of cells, and could be observed at time points in culture from $16 \mathrm{hr}$ to $14 \mathrm{~d}$. The variation in the timetable by which the neurons develop more topographically segregated populations of MAPs is probably due to the neuronal culture system used here, which includes a variety of cell types that have achieved variable levels of maturity at the time of plating. The confinement of the antigen undergoing elimination to the proximal portion of the neurite is consistent with the addition of the MAPs to the distal end of the elongating neurite. In this model, when the neurite attempts to achieve a more uniform population of MAPs, the antigen being eliminated will appear to recede toward the cell body (Fig. 8). A similar distribution of MAP2 within the proximal portion of the developing cerebellar granule cell in culture has been reported by Alaimo-Beuret and Matus (1985). The implication of the distal addition of MAPs and the distal assembly of microtubules is that the MAPs and tubulin must be transported to the region of the growth cone as unassembled elements. Since phosphorylated forms of some MAPs have been shown to be less effective at promoting microtubule assembly 


\section{AXON DEVELOPMENT}

- INITIAL NEURITE EXTENSION

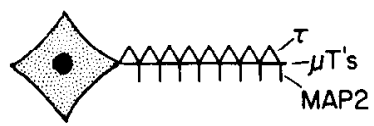

- ELIMINATION OF MAP2

Model 1-Distal Assembly
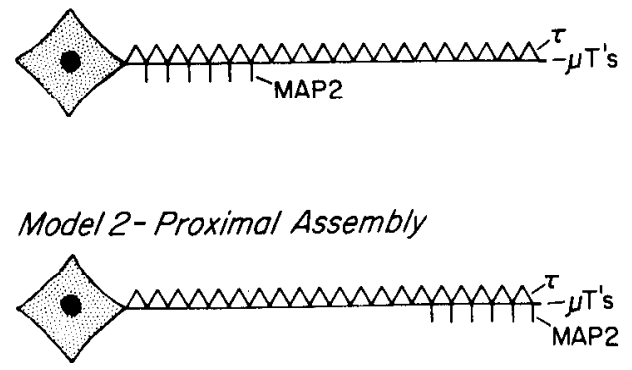

Figure 8. Model for the addition of MAPs to microtubules during the elongation of axon-like neurites in culture. The neurite as it initially extends contains both MAP2 and tau. At some point in development, after the neurite bears some light-microscopic resemblance to an axon, it begins to eliminate MAP2. If microtubules extended from the cell body (proximal assembly), then those microtubules containing MAP2 would appear distally during the transformation process to a more homogeneous population of MAPs in the axon (model 2). If MAPs bound microtubules at the growth cone of an elongating axon, where the microtubules themselves could assemble (distal assembly), then those microtubules containing MAP2 would appear proximally when the transformation process began and, depending on the rate of neurite extension vis-à-vis the anterograde transport of the vestigial MAP2-containing microtubules, might appear to diminish diffusely (model 1 ).

and are quantitatively increased during development, it may be that MAP2 and tau are transported in their phosphorylated forms so as to inhibit polymerization until they reach the region of the growth cone where dephosphorylation triggers microtubule assembly.

\section{References}

Alaimo-Beuret, D., and A. Matus (1985) Changes in the cytoplasmic distribution of microtubule-associated protein 2 during the differentiation of cultured cerebellar granule cells. Neuroscience 14: 11031115.

Bamburg, J. R., D. Bray, and K. Chapman (1986) Assembly of microtubules at the tip of growing axons. Nature 321: 788-790.

Banker, G. A., and W. M. Cowan (1977) Rat hippocampal neurons in dispersed cell culture. Brain Res. 126: 397-425.

Bartlett, W. P., and G. A. Banker (1984a) An electron microscopic study of the development of axons and dendrites by hippocampal neurons in culture. I. Cells which develop without intercellular contacts. J. Neurosci. 4: 1944-1953.

Bartlett, W. P., and G. A. Banker (1984b) An electron microscopic study of the development of axons and dendrites by hippocampal neurons in culture. II. Synaptic relationships. J. Neurosci. 4: 19541965.

Binder, L. I., A. Frankfurter, H. Kim, A. Caceres, M. R. Payne, and L. I. Rebhun (1984) Heterogeneity of microtubule-associated protein 2 during rat brain. Proc. Natl. Acad. Sci. USA. 81: 5613-5617.

Binder, L. I., A. Frankfurter, and L. I. Rebhun (1985) The distribution of tau in the mammalian central nervous system. J. Cell Biol. 101: 1371-1378.

Burgoyne, R. D., and R. Cumming (1984) Ontogeny of microtubule- associated protein 2 in rat cerebellum: Differential expression of the doublet polypeptides. Neuroscience 11: 157-167.

Caceres, A., G. Banker, O. Steward, L. Binder, and M. Payne (1984) MAP2 is localized to the dendrites of hippocampal neurons which develop in culture. Dev. Brain Res. 13: 314-318.

Caceres, A., G. A. Banker, and L. Binder (1986) Immunocytochemical localization of tubulin and microtubulc-associated protcin 2 during the development of hippocampal neurons in culture. J. Neurosci. 6 : 714-722.

Couchie, D., A. Faivre-Bauman, J. Puymirat, J. Guilleminot, A. TixierVidal, and J. Nunez (1986) Expression of microtubule-associated proteins during the early stages of neurite extension by brain neurons cultured in a defined medium. J. Neurochem. 47: 1255-1261.

Crandall, J. E., M. Jacobson, and K. S. Kosik (1986) Ontogenesis of microtubule-associated protein 2 (MAP2) in embryonic mouse cortex. Dev. Brain Res. 28: 127-133.

Drubin, D. G., D. Caput, and M. W. Kirschner (1984) Studies on the expression of the microtubule-associated protein, tau, during mouse brain development, with newly isolated complementary DNA probes. J. Cell Biol. 98: 1090-1097.

Escobar, M. I., H. Pimicnta, V.S. Cavincss, M. Jacobson, J. E. Crandall, and K. S. Kosik (1986) Architecture of apical dendrites in the murine neocortex: Dual apical dendritic systems. Neuroscience 17:975-989.

Francon, J., A. M. Lennon, A. Fellous, A. Mareck, M. Pierre, and J. Nunez (1982) Heterogeneity of microtubule-associated proteins and brain development. Eur. J. Biochem. 129: 465-471.

Galloway, P. G., G. Perry, K. S. Kosik, and P. Gambetti (1987) Hirano bodies contain tau protein. Brain Res. 403: 337-340.

Heidemann, S. R., J. M. Landers, and M. A. Hamborg (1981) Polarity orientation of axonal microtubules. J. Cell Biol. 91: 661-665.

Heriot, K., P. Gambetti, and R. J. Lasek (1985) Proteins transported in slow components a and $\mathrm{b}$ of axonal transport are distributed differently in the transverse plane of the axon. J. Cell Biol. 100: 11671172.

Hirokawa, N., W. A. Glicksman, and M. Willard (1984) Organization of mammalian neurofilament polypeptides within the axonal cytoskeleton. J. Cell Biol. 98: 1523-1536.

Hsu, S. M., L. Rainc, and H. Fanger (1981) The use of avidin-biotinperoxidase complex $(A B C)$ in immunoperoxidase technique: A comparison between $A B C$ and unlabeled antibody (PAP) procedures. J. Histochem. Cytochem. 29: 577-580.

Kosik, K. S., L. K. Duffy, M. M. Dowling, C. Abraham, A. McCluskey, and D. J. Selkoe (1984) Microtubule-associated protein 2: Monoclonal antibodies demonstrate selective incorporation of certain epitopes into Alzheimer neurofibrillary tangles. Proc. Natl. Acad. Sci. USA 81: 7941-7945.

Kosik, K. S. C. L. Joachim, and D. J. Selkoe (1986) Microtubuleassociated protein tau is a major antigenic component of paired helical filaments in Alzheimer's disease. Proc. Nat1. Acad. Sci. IUSA 83: 4044-4048.

Kowall, N. W., and K. S. Kosik (1986) Tau histochemistry reveals widespread cytoskeletal disorganization in Alzheimer's disease. Soc. Neurosci. Abstr. 12: 943.

Kriegstein, A. R., and M. A. Dichter (1983) Morphological classification of rat cortical neurons in cell culture. J. Neurosci. 3: 16341647.

Landis, S. (1977) Morphological properties of the dendrites and axons of dissociated rat sympathetic neurons. Soc. Neurosci. Abstr. 3: 525.

Lawrence, J. B., and R. H. Singer (1986) Intracellular localization of messenger RNAs for cytoskeletal proteins. Cell 45: 407-415.

Mareck, A., A. Fellous, J. Francon, and J. Nunez (1980) Changes in composition and activity of microtubule-associated proteins during brain development. Nature 284: 353-355.

Mates, S. L., and J. S. Lund (1983) Spine formation and maturation of type 1 synapses on spiny stellate neurons in primate visual cortex. J. Comp. Ncurol. 221: 91-97.

Matus, A., R. Bernhardt, and T. Hugh-Jones (1981) High molecular weight microtubule-associated proteins are preferentially associated with dendritic microtubules in brain. Proc. Natl. Acad. Sci. USA 78: 3010-3014.

McCarthy, K. D., and J. de Vellis (1980) Preparation of separate astroglial and oligodendroglial cell cultures from rat cerebral tissue. J. Cell Biol. 85: 890-892.

McLean, I. W., and P. K. Nakane (1974) Periodate-lysine-paraformaldehyde fixative. A new fixative for immunoelectron microscopy. J. Histochem. Cytochem. 22: 1077-1083. 
Miller, P., V. Walter, W. E. Therkauf, R. B. Vallee, and P. DeCamilli (1982) Frozen tissue sections as an experimental system to reveal specific binding sites for the regulatory subunit of type II cAMPdependent protein kinase in neurons. Proc. Natl. Acad. Sci. USA 79: $5562-5566$.

Neale, E. A., R. L. MacDonald, and P. G. Nelson (1978) Intracellular horseradish peroxidase injection for correlation of light and electron microscopic anatomy with synaptic physiology of cultured mouse spinal cord neurons. Brain Res. 152: 265-282.

Peng, I., L. I. Binder, and M. M. Black (1986) Biochemical and immunological analyses of cytoskeletal domains of neurons. J. Cell Biol. 102: 252-262.

Ranscht, B., P. A. Clapshaw, J. Price, M. Noble, and W. Seifert (1982) Development of oligodendrocytes and Schwann cells studied with a monoclonal antibody against galactocerebroside. Proc. Natl. Acad. Sci. USA 79: 2709-2713.
Shaw, G., W. Osborn, and K. Weber (1981) An immunofluorescence microscopical study of the neurofilament triplet proteins, vimentin, and glial fibrillary acidic protein within the adult rat brain. Eur. J. Cell Biol. 26: 68-82.

Towbin, H., T. Staehelin, and J. Gordon (1979) Electrophoretic transfer of proteins from polyacrylamide gels to nitrocellulose sheets: Procedure and some applications. Proc. Natl. Acad. Sci. USA 76:43504354.

Tytell, M., S. T. Brady, and R. J. Lasek (1984) Axonal transport of a subclass of tau proteins: Evidence for the regional differentiation of microtubules in neurons. Proc. Natl. Acad. Sci. USA 81: 1570-1574.

Wakshull, E., M. I. Johnson, and H. Burton (1979) Postnatal rat sympathetic neurons in culture. I. A comparison with embryonic neurons. J. Neurophysiol. 42: 1410-1425. 